Methods In this study, virally suppressed $\mathrm{CHB}$ patients (HBV DNA <LLOQ x 6 months, <20 IU/mL at screening) with moderate or severe RI or with ESRD on $\mathrm{HD}$ at screening while receiving TDF and/or other OAVs for $\geq 48$ weeks were enrolled and switched to TAF for 96 weeks. Safety assessments including adverse events (AEs), changes in bone $\mathrm{BMD}$ and renal $\left(\mathrm{eGFR} \mathrm{CG}_{\mathrm{CG}}\right.$, serum phosphorus serum creatinine - except in ESRD patients) parameters, viral suppression, serological and biochemical responses were serially assessed.

Results Of 93 patients (mod-severe RI 78; ESRD on HD 15 ), most (74\%) were male and Asian (77\%), 51\% $\geq 65 \mathrm{y}$, 83\% HBeAg-negative, 34\% cirrhosis, and median ALT 17 U/L. Up to $24 \%$ had osteoporosis at hip and/or spine, with most having comorbidities. Twelve (13\%; 11 mod-severe RI and 1 ESRD) patients discontinued the study early (5-withdrew consent, 3-deaths [none treatment-related], 2-AE, 2investigator decision). Viral suppression (HBV DNA<20IU/ $\mathrm{mL}$ ) was maintained in all patients remaining on treatment (i.e. missing equals excluded); a high proportion had target not detected. Overall, TAF was well tolerated with no Grade 3,4 or serious AEs related to study treatment. Relative to baseline levels, switching to TAF resulted in small median\% increases in hip/spine BMD in those with moderate to severe RI, and small median decreases in ESRD patients. 2 patients with mod-severe RI had a bone fracture (ankle, rib). Median eGFR $_{\mathrm{CG}}$ increased while urinary markers of proximal tubular function progressively decreased in mod-severe RI patients.

Conclusions Renally-impaired $\mathrm{CHB}$ patients, including ESRD patients on HD, who were switched to TAF from TDF and/or other OAVs maintained high rates of viral suppression, and bone and renal parameters remained stable or slightly improved after 2 years of treatment.

\section{IDDF2021-ABS-0079 SWITCHING FROM TENOFOVIR DISOPROXIL FUMARATE (TDF) AND/OR OTHER ORAL ANTIVIRALS (OAVS) TO TENOFOVIR ALAFENAMIDE (TAF) IN VIRALLY SUPPRESSED CHRONIC HEPATITIS B (CHB) PATIENTS WITH HEPATIC IMPAIRMENT: FINAL 2-YEAR EFFICACY AND SAFETY RESULTS FROM A PHASE 2}

${ }^{1}$ Young-Suk Lim* ${ }^{2}{ }^{2}$ Chun-Yen Lin, ${ }^{3}$ Jeong Heo, ${ }^{4}$ Ho Bae, ${ }^{5}$ Wan-Long Chuang, ${ }^{6}$ Tak Yin Owen Tsang, ${ }^{7}$ Claire Fournier, ${ }^{8}$ Aric Josun Hui, ${ }^{9}$ Huy Trinh, ${ }^{10}$ Carol Yee Kwan Chan, ${ }^{10}$ Susanna K Tan, ${ }^{10}$ Yang Zhao, ${ }^{10}$ John F Flaherty, ${ }^{10}$ Vithika Suri, ${ }^{10}$ Anuj Gaggar, ${ }^{10}$ Dianna M Brainard, ${ }^{11}$ Stephen Ryder, ${ }^{12}$ Harry LA Janssen. ${ }^{1}$ Asan Medical Center, University of Ulsan College of Medicine, Seoul, Korea, South; ${ }^{2}$ Chang Gung University College of Medicine, Taoyuan City, Taiwan; ${ }^{3}$ Pusan National University School of Medicine, Pusan National University Hospital, Busan, Korea, South; ${ }^{4}$ Asian Pacific Liver Center, St. Vincent Medical Center, Los Angeles, California, USA; ${ }^{5}$ Kaohsiung Medical University Chung-Ho Memorial Hospital, Kaohsiung City, Taiwan; ${ }^{6}$ Princess Margaret Hospital, Hong Kong; ${ }^{7}$ Université de Montréal, Québec, Canada; ${ }^{8}$ Alice Ho Miu Ling Nethersole Hospital, Hong Kong; ${ }^{9}$ San Jose Gastroenterology, San Jose, California, USA; ${ }^{10}$ Gilead Sciences, Inc., Foster City, California, USA; ${ }^{11}$ NIHR: Nottingham Biomedical Research Centre, Nottingham University Hospitals NHS Trust, Nottingham, UK; ${ }^{12}$ Toronto Centre for Liver Disease, Toronto General Hospital, University Health Network, Toronto, Ontario, Canada

\subsection{6/gutjnl-2021-IDDF.81}

Background We have previously shown that in hepatically impaired $\mathrm{CHB}$ patients, switching to TAF from TDF and/or other OAVs maintains high rates of viral suppression with stable bone and renal safety parameters through 48 weeks. Here we present our final 2-year (Week 96) results.

Methods In this Phase 2 study (NCT03180619), virally suppressed CHB patients (HBV DNA <LLOQ x 6 months and $<20 \mathrm{IU} / \mathrm{mL}$ at screening) with Child-Turcotte-Pugh (CTP) scores $\geq 7$ and $\leq 12$ at screening (or previously documented to be $\geq 7$ ) while receiving TDF and/or other OAVs for $\geq 48$ weeks were enrolled and switched to TAF $25 \mathrm{mg}$ QD for 96 weeks. Safety assessments including changes in bone (hip and spine BMD) and renal (CrCL by Cockcroft-Gault [eGFR $\mathrm{CG}_{\mathrm{CG}}$, serum creatinine) parameters, viral suppression, and serological and biochemical responses were serially assessed.

Results Of 31 patients enrolled, mean age was 55 y $(29 \%$ $\geq 60$ y), 68\% male, $81 \%$ Asian, and 90\% HBeAg-negative; median (Q1, Q3) CTP and MELD scores were $6(5,8)$ and 10 (7.5, 14.2), respectively, median eGFR CG $_{9} 98.5 \mathrm{~mL} / \mathrm{min}$; $19 \%$ had osteoporosis on spine DXA. Twenty-five (81\%) patients completed 96 weeks of TAF treatment (6 discontinued early: 2-withdrew consent, 1-adverse event [AE; Grade 2 creatinine increase], 1-investigator decision, and 2-death [respiratory failure and aspiration pneumonia - both not treatmentrelated]). Week 96 efficacy/safety results are summarized in the Table. $96 \%$ of patients on TAF treatment had HBV DNA $<20 \mathrm{IU} / \mathrm{mL}$ with a high proportion having normal ALT levels. Bone and renal parameters remained stable. TAF was well tolerated with no patients having a Grade 3 or $4 \mathrm{AE}$ or a serious AE related to treatment.

Conclusions At 2 years, $\mathrm{CHB}$ patients with hepatic impairment who were switched to TAF maintained high rates of viral suppression and normal ALT values while bone and renal parameters remained stable.

\begin{tabular}{|l|l}
\hline IDDF2021-ABS-0080 & 96-WEEK EFFICACY AND SAFETY OF \\
& TENOFOVIR DISOPROXIL FUMARATE (TDF) \\
& TO TENOFOVIR ALAFENAMIDE (TAF) \\
& SWITCH VS. CONTINUED TDF TREATMENT \\
& AMONG VIROLOGICALLY-SUPPRESSED \\
& HEPATITIS B PATIENTS OF ASIAN \\
& ETHNICITY
\end{tabular}

${ }^{1}$ Sang Hoon Ahn*, ${ }^{2}$ Jia-Horng Kao, ${ }^{3}$ Pietro Lampertico, ${ }^{4}$ Alnoor Ramij, ${ }^{5}$ Scott Fung, ${ }^{6}$ WanLong Chuang, ${ }^{7}$ Yoon Jun Kim, ${ }^{8} \mathrm{Chi}-Y_{i}$ Chen, ${ }^{9}$ Edward Tam, ${ }^{10} \mathrm{Ho}$ Bae, ${ }^{11}$ Xiaoli Ma, ${ }^{12}$ June Sung Lee, ${ }^{13}$ Carol Yee Kwan Chan, ${ }^{13}$ Leland I Yee, ${ }^{13}$ Shalini Sethi, ${ }^{13}$ John Flaherty, ${ }^{13}$ Yang Zhao, ${ }^{13}$ Anuj Gaggar, ${ }^{14} \mathrm{Hie}$ Won Hann, ${ }^{15}$ Young-Suk Lim, ${ }^{16} \mathrm{Henry}$ Lik Yuen Chan. ${ }^{1}$ Severance Hospital, Yonsei University College of Medicine, Seoul, Korea, South; ${ }^{2}$ Division of Gastroenterology and Hepatology, Department of Internal Medicine, National Taiwan University Hospital, Taipei, Taiwan; ${ }^{3}$ Foundation IRCCS Ca' Granda Ospedale Maggiore Policlinico, Division of Gastroenterology and Hepatology, Milan, Italy. CRC 'A. M. and A. Migliavacca' Center for Liver Disease, Department of Pathophysiology and Transplantation, University of Milan, Milan, Italy; ${ }^{4}$ Gastrointestinal Research Institute (GIRI), Vancouver, Canada; ${ }^{5}$ Toronto Centre for Liver Disease, Toronto General Hospital, University Health Network, Toronto, Canada; ${ }^{6}$ Hepatobiliary Division, Department of Internal Medicine, Kaohsiung Medical University Hospital, Kaohsiung Medical University, Kaohsiung, Taiwan; ${ }^{7}$ Seoul National University Hospital, Seoul, Korea, South; ${ }^{8}$ Division of Gastroenterology and Hepatology, Department of Medicine, Ditmanson Medical Foundation Chiayi Christian Hospital, Chiayi, Taiwan; ${ }^{9}$ Liver and Intestinal Research Centre, Vancouver, Canada; ${ }^{10}$ Asian Pacific Liver Center, St. Vincent Medical Center, Los Angeles, USA; ${ }^{11}$ Hahnemann University Hospital, Philadelphia, PA, USA; ${ }^{12}$ Department of Internal Medicine, Inje University Ilsan Paik Hospital, Goyang, Korea, South; ${ }^{13}$ Gilead Sciences, Foster City, CA, USA; ${ }^{14}$ Thomas Jefferson University Hospital, Philadelphia, Pennsylvania, USA; ${ }^{15}$ Asan Medical Center, University of Ulsan College of Medicine, Seoul, Korea, South; ${ }^{16}$ The Chinese University of Hong Kong, Hong Kong

10.1136/gutjnl-2021-IDDF.82 
Background Study 4018, an international Phase 3 study, previously demonstrated switching to tenofovir alafenamide (TAF) vs continued tenofovir disoproxil fumarate (TDF) in suppressed chronic hepatitis $\mathrm{B}(\mathrm{CHB})$ patients has non-inferior efficacy (TAF vs TDF) with superior bone/renal safety. We analysed the efficacy and safety of switching in Asian Ethnicity (AE) patients in Study 4018.

Methods CHB patients on TDF for $\geq 48$ weeks with HBV DNA less than LLOQ for $\geq 20 \mathrm{IU} / \mathrm{ml}$ at screening were randomized to TAF $25 \mathrm{mg}$ QD or TDF $300 \mathrm{mg}$ QD, each with a matching placebo, and treated for 48 weeks in a double-blind (DB) fashion followed by all patients receiving open-label (OL) TAF $25 \mathrm{mg}$ QD for an additional 48 weeks.

Results 400/488 (82\%) were AE patients who received at least 1 dose of study drug, with 195 in the TAF-TAF arm ad 205 in the TDF-TAF arm. Virologic suppression was similarly maintained at 96-wk in both groups (95\%: TAF and 94\% TDF). Higher percent changes in BMD from baseline (both hip and spine) were seen among the TAF-TAF group [1.2 (3.0 SD) for hip and 2.5 (4.0) SD for spine] vs TDF-TAF group [0.12 (2.8 SD) for hip and 1.5 (3.8) SD for spine]. eGFR ${ }_{\mathrm{CG}}$ increased over years in the TAF-TAF group and from Week 48 to 96 in the TDF-TAF group. There were no study drugrelated treatment-emergent grade 3 or 4 AEs or SAEs.

Conclusions Following TDF to TAF switch, viral suppression was maintained, with improved bone and renal safety parameters through 96 weeks in $\mathrm{AE}$ patients.

\section{IDDF2021-ABS-0083 ASSESSMENT PRE- AND POST-TREATMENT QUALITY OF LIFE BY EORTC QLQ-30 QUESTIONNAIRE IN PATIENTS WITH HEPATOCELLULAR CARCINOMA AT HANOI MEDICAL UNIVERSITY, VIETNAM}

Thuy Trang Ha*, Minh Duc Pham, Ngoc Anh Trang. Ha Noi Medical University, Vietnam

\subsection{6/gutjnl-2021-IDDF.83}

Background To assess the quality of life (QoL) of patients with hepatocellular carcinoma (HCC) by the European Organization for the Research and Treatment of Cancer quality of life questionnaire (EORTC QLQ- C30).

Methods A prospective study was conducted from August 2020 to August 2021. We evaluated the QoL of 30 patients diagnosed of HCC at Bach Mai and Hanoi Medical University Hospital after a month of treatment. EORTC-QLQ C30 items were converted to scores according to an available formula, the total score for each section ranged from 0 to 100 . A low functional score reflected a low QoL, while a lower symptom score indicated a higher QoL. The overall EORTC score (C30 index score) would be calculated according to the available formula. The lower the C30 index score was, the better the QoL would be.

Results The C30 index before transarterial chemoembolization (TACE) assessed by the EORTC QLQ- C30 questionnaire was $27.344 \pm 10.15$ and that after TACE was $19.63 \pm 11.3$; the difference was statistically significant with $\mathrm{p}$ value $<$ 0.0001. The pre-and post-TACE overall EORTC QLQ- C30 score, the functional and symptoms score had a statistically significant difference $(p$ value $<0.05)$. The QoL score had a positive correlation with the degree of liver failure according to Child-Pugh classifications and ECOG PS grade with rho index of $0.401(\mathrm{p}=0.028<0.05)$ and $0.682(\mathrm{p}<0.001)$, respectively. The EORTC QLQ-C questionnaire was useful in assessing the QoL of HCC patients with pre-and post-treatment Cronbach's alpha scores of 0.816 and 0.920, respectively.

Conclusions The EORTC QLQ- C30 questionnaire was a useful scale in evaluating the pre-and post-treatment quality of life in patients with HCC, which correlates with the patient's physical condition and liver function.

\section{IDDF2021-ABS-0084 TUBERCULOSIS AS A COMMON CAUSE OF POST-LIVER TRANSPLANT ASCITES}

TR Sowmya*. AlG Hospitals, Hyderabad, Telangana State, India

\subsection{6/gutjnl-2021-IDDF.84}

Background Refractory ascites due to cirrhosis is one of the common indications for liver transplant (LT). Ascites are expected to resolve within 2-4 weeks following orthotopic liver transplant as the reversal of hemodynamic changes occurs by then. New-onset ascites after transplant has varied causes. The most common causes for the development of ascites after LT are hepatic inflow or outflow obstructions, hepatitis C, graft rejection, prolonged cold ischemia time. In addition, extrahepatic causes like infections, chronic kidney diseases, or heart failure can lead to ascites.

Methods In this retrospective study, we aimed to assess the incidence and causes of ascites in post-transplant patients during the last year.

Results A total of 95 patients underwent living donor liver transplantation (LDLT) in our institution. Seven out of 95 developed ascites post-liver transplant. The mean age of patients was $46 \pm 18.98$ years. $57 \%$ of patients were females. The indication of LDLT was decompensated cirrhosis in all patients. Three underwent LDLT for AUD-related liver disease, one each for NASH, chronic hepatitis B, Budd-Chiari syndrome, and Wilson disease. The mean time to ascites onset was 2.9 months. All the patients had ascites prior to liver transplant, out of which 5 (71\%) had refractory ascites. $5(71 \%)$ had HRS prior to transplant. Cold ischemia time was $98 \pm 19 \mathrm{~min}$ (IDDF2021-ABS-0084 Table 1). Five patients were diagnosed with tubercular ascites, one had tacrolimusrelated proteinuria and ascites formation, and one more patient developed IgA nephropathy causing significant proteinuria and ascites. None of the patients had concomitant pulmonary tuberculosis. Rifampicin, levofloxacin, and ethambutol were initiated with a gradual introduction of isoniazid over the next 15 days. One of the patients developed ATT-induced liver injury and graft dysfunction after switching from rifampicin to rifabutin. He was treated with $\mathrm{N}$-acetylcysteine and had delayed recovery with conservative management.

Conclusions Tuberculosis is a common cause of post-transplant ascites. Although global data suggest vascular etiologies as a common cause of persistent ascites post-transplant, in the Indian scenario, tuberculosis should always be considered as a differential diagnosis while evaluating these patients. Being a potentially curable cause, it significantly improves graft survival after treatment. 